\title{
Exponential Synchronization of the Hopfield Neural Networks with New Chaotic Strange Attractor
}

\author{
Zhan-Ji GUI, Kai-Hua WANG* \\ Department of Software Engineering, Hainan College of Software Technology ,qionghai, 571400, P.R. China \\ School of Mathematics and Statistics, Hainan Normal University, Haikou, Hainan, 571158, P.R. China \\ *Corresponding author: kaihuawang@qq.com
}

\begin{abstract}
This paper studies the problems of exponential synchronization for the Hopfield neural networks with impulsive effects and Gui chaotic strange attractor. By employing the Lyapunov functional method of impulsive functional differential equations, some criteria for synchronization between two impulsive neural networks are derived. An illustrative example is provided to show the effectiveness and feasibility of the proposed method and results.
\end{abstract}

\section{Introduction}

Recently, the issue of controlled synchronization in complex dynamical networks has become a rather significant topic in both theoretical research and practical applications [1-3]. It is known that in theory and practice, impulsive control has been widely used to stabilize and synchronize chaotic systems. For example, Yang and Chua derived some sufficient conditions for the stabilization and synchronization of Chua's oscillators via impulsive control[4]; Xie, Wen and $\mathrm{Li}$ obtained sufficient conditions for the stabilization and synchronization of the Lorenz system via impulsive control with varying impulsive intervals[5]. The Hopfield neural networks with impulse effect are studied, where the criteria on the existence, uniqueness and global stability of periodic solution are obtained. Further, Gui chaos strange attractor was also found[6-10].

Motivated by the above discussions, the aim of this paper is to study the synchronization of Hopfield neural networks with a Gui chaotic strange attractor. By employing the Lyapunov-like stability theory of impulsive functional differential equations, some criteria for synchronization of Hopfield neural networks are derived.

The remainder of the paper is organized as follows: Section 2 describes the issue of synchronization of coupled impulsive systems with a Gui chaotic strange attractor. In Section 3, some sufficient conditions for the synchronization are derived by constructing suitable Lyapunov-like function. In Section 4, an illustrative example is given to show the effectiveness of the proposed method. Conclusions are given in Section 5.

\section{Preliminaries Formulation}

In this paper, we consider the following nonautonomous Hopfield neural networks model with impulses

$$
\left\{\begin{array}{c}
\dot{x}_{i}=-a_{i} x_{i}(t)+\sum_{j=1}^{n} b_{i j} f_{j}\left(x_{j}(t)\right)+c_{i}(t), \\
t>0, t \neq t_{k}, \\
\Delta x_{i}\left(t_{k}\right)=x_{i}\left(t_{k}^{+}\right)-x_{i}\left(t_{k}^{-}\right)=-\gamma_{i k} x_{i}\left(t_{k}\right), \\
t>0, t=t,
\end{array}\right.
$$

where $\Delta x_{i}\left(t_{k}\right)=x_{i}\left(t_{k}^{+}\right)-x_{i}\left(t_{k}^{-}\right)$are the impulses at moments $t_{k}^{+}$and $t_{1}<t_{2}<\cdots$ is a strictly increasing sequence such that $\lim _{k \rightarrow \infty} t_{k}=+\infty ; x_{i}(t)$ corresponds to the state of the $i$ th unit at time $t, a_{i}$ is a positive constant set; $b_{i j}$ denotes the strength of the $j$ th unit on the $i$ th unit at time $t, c_{i}(t)$ denotes the external input on the $i$ th neuron and $f_{j}\left(x_{j}(t)\right)$ denotes the output of the $j$ th unit at time $t$.

As usual in the theory of impulsive differential equations, at the points of discontinuity $t_{k}$ of the solution $t \rightarrow x_{i}(t)$ we assume that $x_{i}\left(t_{k}\right) \equiv x_{i}\left(t_{k}^{-}\right)$. It is clear that, in general, the derivatives $x_{i}^{\prime}\left(t_{k}\right)$ do not exist. On the other hand, according to the first equality of (1) there exist the limits $x_{i}^{\prime}\left(t_{k_{k}}^{\mp}\right)$. 
According to the above convention, we assume $x_{i}^{\prime}\left(t_{k}\right) \equiv x_{i}^{\prime}\left(t_{k}^{-}\right)$.

Throughout this paper, we assume that:

(H1) Functions $f_{j}(u)$ satisfy the Lipschitz condition, i.e., there are constants $L_{j}>0$ such that

$$
\left|f_{j}\left(u_{1}\right)-f_{j}\left(u_{2}\right)\right| \leq L_{j}\left|u_{1}-u_{2}\right|
$$

for all $u_{1}, u_{2} \in R=(-\infty,+\infty)$.

$\left(\mathbf{H}_{2}\right)$ There exists a positive integer $p$, such that

$$
t_{k+p}=t_{k}+\omega, \gamma_{i(k+p)}=\gamma_{i k}
$$

$i=1,2, \cdots, n, k=1,2, \cdots$

(H3) $a_{i}>0, c_{i}(t)$ are all continuous $\omega$-periodic functions.

As is known to all that (1) can exhibit chaotic phenomenons ${ }^{[6-10]}$. In order to show it clearly, we give the following example.

Example 1. Consider a two-dimensional neural network with impulsive effects, which can be described by the following impulsive differential equations:

$$
\left\{\begin{aligned}
& \dot{x}_{1}=-2 x_{1}(t)+0.56 f_{1}\left(x_{1}(t)\right)+0.48 f_{2}\left(x_{2}(t)\right) \\
&+c_{1}(t), \quad t>0, t \neq n T \\
& \dot{x}_{2}=-2 x_{2}(t)+0.61 f_{1}\left(x_{1}(t)\right)+0.3 f_{2}\left(x_{2}(t)\right) \\
&+c_{2}(t), \quad t>0, t \neq n T \\
& x_{1}\left(n T^{+}\right)=\left(1-\gamma_{1}\right) x_{1}(n T), n=1,2, \cdots \\
& x_{2}\left(n T^{+}\right)=\left(1-\gamma_{2}\right) x_{2}(n T), n=1,2, \cdots
\end{aligned}\right.
$$

where $f_{j}\left(x_{j}\right)=0.5\left(\left|x_{j}+1\right|-\left|x_{j}-1\right|\right)$. Obviously, $f_{j}(x)$ satisfy $\left(\mathrm{H}_{1}\right)$. Now we investigate the influence of the period $\mathrm{T}$ of impulsive effect on the system (2). Set

$$
\begin{gathered}
c_{1}(t)=2-0.5 \cos (1.5 t), \\
c_{2}(t)=2+0.5 \sin (0.5 t), \\
T=0.4 \pi, \gamma_{1}=0.35, \gamma_{2}=0.2 .
\end{gathered}
$$

According to Theorems 1 and 2 in [6], the cellular neural network model (2) has a unique $2 \pi$-periodic solution with 5 -impulses in a period.

Furthermore, if $T=1$, then $\left(\mathrm{H}_{2}\right)$ isn't satisfied. Periodic oscillation of system (2) will be destroyed by impulses effect. Numeric results show that system (2) still has a global attractor which can be a Gui chaotic strange attractor. Every solutions of system (2) will finally tend to the new chaotic strange attractor which is useful in exponential synchronization (see Fig.1-3).

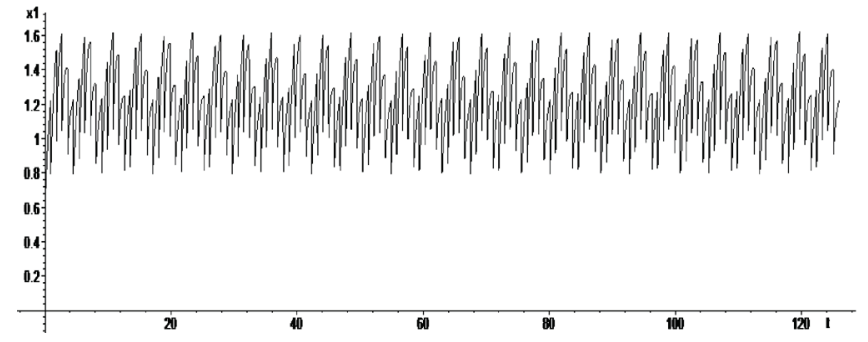

Figure 1. Time-series of the $x_{1}(t)$ of system (2) with $T=1$

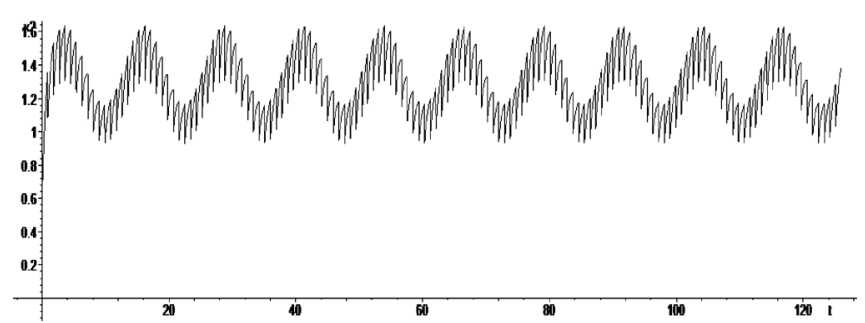

Figure 2. Time-series of the $x_{2}(t)$ of system (2) with $T=1$

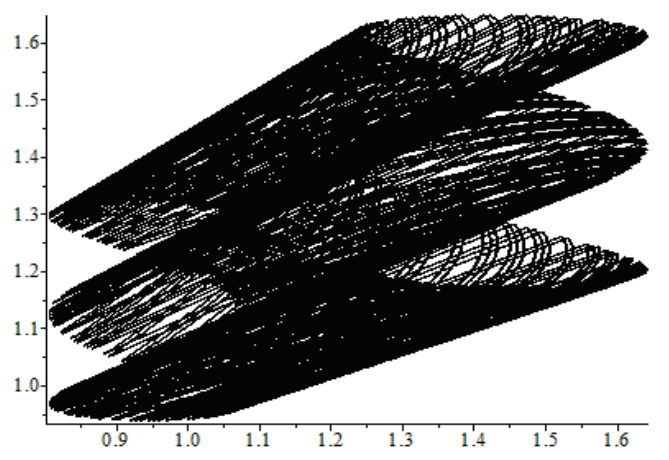

Figure 3. Phase portrait of Gui chaotic strange attractor of system (2)

For the purpose of synchronization, we introduce the response system that is driven by (1) via a set of signals

$$
\left\{\begin{array}{c}
\dot{y}_{i}=-a_{i} y_{i}(t)+\sum_{j=1}^{n} b_{i j} f_{j}\left(y_{j}(t)\right)+c_{i}(t), \\
t>0, t \neq t_{k} \\
y_{i}\left(t_{k}^{+}\right)=x_{i}\left(t_{k}^{-}\right)-\gamma_{i k} y_{i}\left(t_{k}^{-}\right) \\
i=1,2, \cdots, n, k=1,2, \cdots
\end{array}\right.
$$

Let $e_{i}(t)=y_{i}(t)-x_{i}(t)$ be the synchronization error, $x_{i}(t)$ and $y_{i}(t)$ are the state variables of drive system (1) and response system (3). The error system of the impulsive synchronization is given by 


$$
\left\{\begin{array}{l}
\dot{e}_{i}=-a_{i} e_{i}(t)+\sum_{j=1}^{n} b_{i j}\left(e_{j}(t)\right), \quad t>0, t \neq t_{k}, \\
e_{i}\left(t_{k}^{+}\right)=-\gamma_{i k} e_{i}\left(t_{k}^{-}\right), \quad i=1,2, \cdots, n, k=1,2, \cdots,
\end{array}\right.
$$

where $g_{j}\left(e_{j}(t)\right)=f_{j}\left(y_{j}(t)\right)-f_{j}\left(x_{j}(t)\right)$. Note that the origin is the equilibrium point of system (4). If $e_{i}(t)$ tends exponentially to origin in evolution, exponential synchronization between two systems would be realized. Our aim is to find some criteria on the impulsive gains $\gamma_{i k}$ such that drive system (1) and respond system (3) are exponentially synchronized for any initial condition.

According to the assumption $\left(\mathrm{H}_{1}\right), g_{i}(\cdot)$ possesses the following properties:

$$
\begin{aligned}
& \left|g_{j}\left(e_{j}(t)\right)\right| \leq L_{j}\left|e_{j}(t)\right|, \quad \text { and } \\
& g_{j}(0)=0, \quad j=1,2 \ldots, n .
\end{aligned}
$$

Throughout the paper, we denote

$$
\|y(t)-x(t)\|=\sum_{j=1}^{n}\left|y_{j}(t)-x_{j}(t)\right|^{r} .
$$

Definition 1. Systems (1) and (3) are said to be exponentially synchronized if there exist constants $M \geq 1$ and $\eta>0$ such that

$$
\|y(t)-x(t)\|=M\|y(0)-x(0)\| e^{-\eta t},
$$

for any $t \geq 0$. Constant $\eta$ is said to be the degree of exponential synchronization.

Lemma 1. Assume that $x>0, y>0, p>1, q>1$, $1 / p+1 / q=1$, then the inequality

$$
x y \leq \frac{1}{p} x^{p}+\frac{1}{q} x^{q}
$$

holds (the inequality is called as Young inequality).

\section{$3 \quad$ Main results}

In this section, we investigate the exponential synchronization of system (1) and (3) by using Lyapunov like functional method.

Theorem 1. Under the assumption $\left(\mathbf{H}_{1}\right),\left(\mathbf{H}_{2}\right)$ and $\left(\mathbf{H}_{3}\right)$, the system (1) and (3) are exponentially synchronized, if there exist positive constants $r>1, w_{i}>0$ and $\sigma_{i j} \in R$ $i, j=1,2, \cdots, n$. such that

$$
\begin{aligned}
& -r a_{i}+(r-1) \sum_{j=1}^{n} L_{j}^{r\left(1-\sigma_{i j}\right) /(r-1)}\left|b_{i j}\right|^{r\left(1-\sigma_{i j}\right) /(r-1)} \\
& +\sum_{j=1}^{n} \frac{\lambda_{j}}{\lambda_{i}} L_{i}^{r \sigma_{j i}}\left|b_{j i}\right|^{r \sigma_{j i}}<0, \quad i=1,2, \ldots, n .
\end{aligned}
$$

$$
\begin{aligned}
& r\left(\varepsilon-a_{i}\right)+(r-1) \sum_{j=1}^{n} L_{j}^{r\left(1-\sigma_{i j}\right) /(r-1)}\left|b_{i j}\right|^{r\left(1-\sigma_{i j}\right) /(r-1)} \\
& +\sum_{j=1}^{n} \frac{\lambda_{j}}{\lambda_{i}} L_{i}^{r \sigma_{j i}}\left|b_{j i}\right|^{r \sigma_{j i}}<0, \quad i=1,2, \ldots, n .
\end{aligned}
$$

Consider the following Lyapunov function:

$$
V(e(t))=\sum_{i=1}^{n} \lambda_{i}\left|e_{i}(t)\right|^{r} e^{r \varepsilon t}
$$

Calculating the upper right dini-derivative $D^{+} V$ of $V$ along the solution of system (4) at the continuous points $t \neq t_{k}, t \geq 0$, we have

$$
\begin{aligned}
D^{+} V(e(t))= & \sum_{i=1}^{n} \lambda_{i}\left[r \varepsilon e^{r \varepsilon t}\left|e_{i}(t)\right|^{r}+r e^{r \varepsilon t}\left|e_{i}(t)\right|^{r-1} .\right. \\
& \left.D^{+}\left|e_{i}(t)\right|\right] \\
= & \sum_{i=1}^{n} \lambda_{i}\left[r \varepsilon e^{r \varepsilon t}\left|e_{i}(t)\right|^{r}+r \operatorname{sign}\left(e_{i}(t)\right) e^{r \varepsilon t} .\right. \\
& \left.\left|e_{i}(t)\right|^{r-1}\left(-a_{i} e_{i}(t)+\sum_{j=1}^{n} b_{i j} g_{j}\left(e_{j}(t)\right)\right)\right] \\
& \left.+r \sum_{j=1}^{n}\left|b_{i j}\right|\left|e_{i}(t)\right|^{r-1}\left|g_{j}\left(e_{j}(t)\right)\right|\right] .
\end{aligned}
$$

By Lemma 1, we have the following inequalities:

$$
\begin{aligned}
& D^{+} V(e(t)) \leq \sum_{i=1}^{n} \lambda_{i} e^{r \varepsilon t}\left[r\left(\varepsilon-a_{i}\right)\left|e_{i}(t)\right|^{r}\right. \\
& +(r-1) \sum_{j=1}^{n} L_{j}^{r\left(1-\sigma_{i j}\right) /(r-1)}\left|b_{i j}\right|^{r\left(1-\sigma_{i j}\right) /(r-1)}\left|e_{i}(t)\right|^{r} \\
& \left.+\sum_{j=1}^{n} L_{j}^{r \sigma_{i j}}\left|b_{i j}\right|^{r \sigma_{i j}}\left|e_{j}(t)\right|^{r}\right] \\
& =\sum_{i=1}^{n} \lambda_{i}\left[r\left(\varepsilon-a_{i}\right)+(r-1) \sum_{j=1}^{n} L_{j}^{r\left(1-\sigma_{i j}\right) /(r-1)} .\right. \\
& +\left.b_{i j}\right|^{r\left(1-\sigma_{i j}\right) /(r-1)} \\
& \left.+\sum_{j=1}^{n} \frac{\lambda_{j}}{\lambda_{i}} L_{i}^{r \sigma_{j i}}\left|b_{j i}\right|^{r \sigma_{j i}}\right]\left|e_{i}(t)\right|^{r} e^{r \varepsilon t} \leq 0, \\
& t \geq 0, t \neq t_{k} .
\end{aligned}
$$

Also, we can calculate right limits of Lyapunov-like function $V(e(t))$ at impulsive moments $t_{k}$ as follows

Proof. By inequality (5), choose a small $\varepsilon>0$ such that 


$$
\begin{aligned}
V\left(e\left(t_{k}^{+}\right)\right) & =\sum_{i=1}^{n} \lambda_{i}\left|e_{i}\left(t_{k}^{+}\right)\right|^{r} e^{r \varepsilon t_{k}^{+}} \\
& =\sum_{i=1}^{n}\left|1-\lambda_{i}\right|\left|e_{i}\left(t_{k}\right)\right|^{r} e^{r \varepsilon t_{k}} \\
& \leq \sum_{i=1}^{n} \lambda_{i}\left|e_{i}\left(t_{k}\right)\right|^{r} e^{r \varepsilon t_{k}}=V\left(e\left(t_{k}\right)\right),
\end{aligned}
$$

which implies that

$$
\begin{aligned}
V(e(t)) & \leq V(e(0))=\sum_{i=1}^{n} \lambda_{i}\left|e_{i}(0)\right|^{r} \\
& =\sum_{i=1}^{n} \lambda_{i}\left|y_{i}(0)-x_{i}(0)\right|^{r} \\
& \leq \max _{1 \leq i \leq n}\left\{\lambda_{i}\right\}\|y(0)-x(0)\|, \\
& \text { for } \quad t \geq 0 .
\end{aligned}
$$

According to (6), we have

$$
\begin{aligned}
V(e(t)) & \geq \min _{1 \leq i \leq n}\left\{\lambda_{i}\right\} \sum_{i=1}^{n}\left|e_{i}(t)\right|^{r} e^{r \varepsilon t} \\
& \geq \min _{1 \leq i \leq n}\left\{\lambda_{i}\right\} e^{r \varepsilon t}\|y(t)-x(t)\| .
\end{aligned}
$$

From $(7)-(8)$, we can obtain

$$
\|y(t)-x(t)\| \leq M\|y(0)-x(0)\| e^{-r \varepsilon t} .
$$

where $M=\max _{1 \leq i \leq n}\left\{\lambda_{i}\right\} / \min _{1 \leq i \leq n}\left\{\lambda_{i}\right\} \geq 1$. According to Definition 1, we conclude that the drive system (1) and the response system (3) are exponentially synchronized. This completes the proof.

\section{$4 \quad$ A Simulation Example}

In this section, we give an example to illustrate the effectiveness of the results obtained in the previous sections. Consider a two-dimensional neural networks with impulsive effects.

Taking (2) as the drive system in Example 1. The response system is constructed as follows:

$$
\left\{\begin{aligned}
& \dot{y}_{1}=-2 y_{1}(t)+0.56 f_{1}\left(y_{1}(t)\right) \\
&+0.48 f_{2}\left(y_{2}(t)\right)+c_{1}(t), \quad t \neq n T, \\
& \dot{y}_{2}=-2 y_{2}(t)+0.61 f_{1}\left(y_{1}(t)\right) \\
&+0.3 f_{2}\left(y_{2}(t)\right)+c_{2}(t), \quad t \neq n T, \\
& y_{1}\left(n T^{+}\right)=x_{1}\left(n T^{-}\right)-\gamma_{1} y_{1}(n T), n=1,2, \cdots, \\
& y_{2}\left(n T^{+}\right)=x_{2}\left(n T^{-}\right)-\gamma_{2} y_{2}(n T), n=1,2, \cdots
\end{aligned}\right.
$$

Then the error system of drive system (2) and respond system (9) is constructed as follows:

$$
\left\{\begin{array}{l}
\dot{e}_{1}=-2 e_{1}(t)+0.56 g_{1}\left(e_{1}(t)\right)+0.48 g_{2}\left(e_{2}(t)\right), \\
\dot{e}_{2}=-2 e_{2}(t)+0.61 g_{1}\left(e_{1}(t)\right)+0.3 g_{2}\left(e_{2}(t)\right), \\
\quad t \neq n T, \\
e_{1}\left(n T^{+}\right)=-\gamma_{1} e_{1}\left(n T^{-}\right), \quad n=1,2, \cdots, \\
e_{2}\left(n T^{+}\right)=-\gamma_{2} e_{2}\left(n T^{-}\right), \quad n=1,2, \cdots .
\end{array}\right.
$$

Numeric results show that system (9) still has a Gui chaotic strange attractor ${ }^{[6-10]}$. The phase plot of Gui chaotic strange attractor of response system is shown in Fig. 4. It is easy to check the conditions in Theorem 1 are satisfied. Therefore, systems (2) and (9) exhibit exponential synchronization. Define $e_{i}(t)=y_{i}(t)-x_{i}(t)$ and the errors (10) between systems (2) and (9) are depicted in Fig. 5.

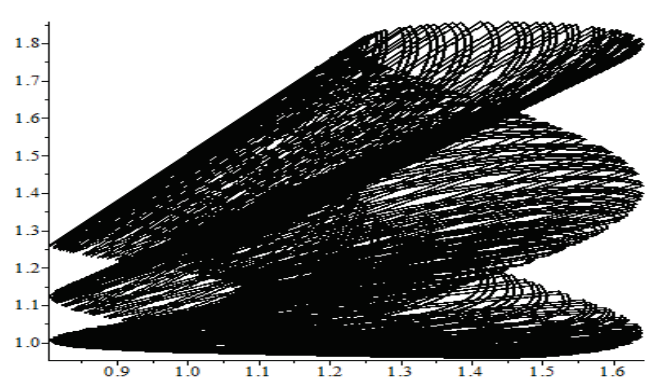

Figure 4. Phase portrait of Gui chaotic strange attractor of system (9)

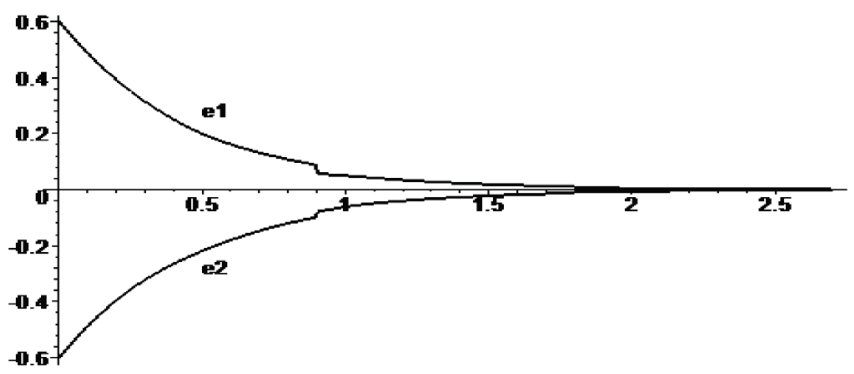

Figure 5. Synchronization errors between drive system (2) and response system (9)

\section{Conclusions}

In this paper, the conditions for the exponential synchronization of a class of neural networks with impulsive effects are derived by utilizing Lyapunov functional method. A numerical simulation is given to show the effectiveness and feasibility of the proposed method. As far as we know, there is no paper to deal with such a problem. 


\section{Acknowledgments}

This research was jointly supported by the Nature Science Foundation of Hainan Province under Grant No. 117164, 117097 and Foundation of Hainan Educational Committee under Grant No. Hnky2017ZD-10.

\section{References}

[1] T. Hayakawa, W.M. Haddad, K.Y. Volyanskyy. Neural network hybrid adaptive control for nonlinear uncertain impulsive dynamical systems, Nonlinear Anal. Hybrid Syst. 2(2008):862-874.

[2] G. Agranovich, E. Litsyn, A. Slavova. Impulsive control of a hysteresis cellular neural network model, Nonlinear Anal. Hybrid Syst. 3(2009):65-73.

[3] J. Zhou, L. Xiang, Z.R. Liu. Synchronization in complex delayed dynamical networks with impulsive effects, Physica A. 384(2007):684-692.

[4] T. Yang, L.O. Chua . Impulsive stabilization for control and synchronization of chaotic system, IEEE Trans. Circuits Syst. I. 44(1997):976-988.

[5] W. Xie, C. Wen, Z. Li.Impulsive control for the stabilization and synchronization of Lorenz system, Phys. Lett. A. 275(2000): 67-72.

[6] Z.J. Gui, W.G. Ge. Existence and uniqueness and periodic solutions of nonautonomous cellular neural networks with impulses, Phys. Lett. A. 354(2006): 8494.

[7] J. Zhang, Z.J. Gui. Existence and stability of periodic solutions of high-order Hopfield neural networks with impulses and delays. Journal of Computational and Applied Mathematics. 224(2)(2009): 602-613.

[8] J. Zhang, Z.J. Gui. Periodic solutions of nonautonomous cellular neural networks with impulses and delays. Nonlinear Analysis: Real World Applications, 10(3)(2009):1891-1903

[9] Z.J. Gui, W.G. Ge. Periodic solution and chaotic strange attractor for shunting inhibitory cellular neural networks with impulses. In Chaos. 16 (2006): 33116-110.

[10] Z.J. Gui, W.G. Ge. Impulsive effect of continuous time neural networks under pure structural variations.In International Journal of Bifurcation and Chaos. 17(2007): 2127-2139. 